\title{
HARDY INEQUALITIES WITH REMAINDER TERMS FOR THE GENERALIZED BAOUENDI-GRUSHIN VECTOR FIELDS
}

\author{
Jingbo Dou, QianQiao Guo And PENGCHENG NiU
}

Abstract. Based on the properties of vector fields and the generalized divergence formula, we prove the Hardy inequalities with remainder terms for the generalized Baouendi-Grushin vector fields and determine the best constants in these Hardy inequalities. Mathematics subject classification (2010): 26D10, 35J70, 35P30.

Keywords and phrases: Generalized Baouendi-Grushin vector field, Hardy inequality, best constant.

\section{REFERENCES}

[1] G. Barbatis, S. Filippas And A. Tertikas, A unified approach to improved $L^{p}$ Hardy inequalities with best constants, Trans. Amer. Math. Soc., 356 (2004), 2169-2196.

[2] G. Barbatis, S. Filippas And A. Tertikas, Series expansion for $L^{p}$ Hardy inequalities, Indiana Univ. Math. J., 52 (2003), 171-190.

[3] W. Beckner, On the Grushin operator and hyperbolic symmetry, Proc. Amer. Math. Soc., 129 (2001), 1233-1246.

[4] L. D'Ambrosio, Hardy inequalities related to Grushin type operators, Proc. Amer. Math. Soc., 132, 3 (2004), 725-734.

[5] L. D'Ambrosio, Hardy type inequalities related to second order degenerate differential operators, Ann. Scuola Norm. Sup. Pisa Cl. Sci., ser. 5, IV (2005), 451-486.

[6] J. Dou, P. NIU, AND Z.YUAN, A Hardy Inequality with Remainder Terms in the Heisenberg Group and the Weighted Eigenvalue Problem, J. Inequ. Appl. 2007(2007), Article ID 32585, 24 pages.

[7] B. FRANCHI AND E. LANCONELLI, Une metrique associée à une classe d'opérateurs elliptiques dégénérés, Proceedings of the meeting Linear partial and pseudodifferential operators, Rend. Sem. Mat. Univ. e Politec. Torino, (1983), 105-114.

[8] B. FRANCHI AND E. LANCONELLI, Hölder regularity theorem for a class of linear nonuniformly elliptic operators with measurable coefficients, Annali Scuola Normale Sup. Pisa, Cl. Sci. (A) IV. Ser. 10 (1983), 523-541.

[9] B. FRANCHI AND E. LANCONELLI, An embedding theorem for Sobolev spaces related to non-smooth vector fields and Harnack inequality, Comm. P. D. E., 9 (1984), 1237-1264.

[10] J. García Azorero, And I. Peral, Hardy inequality and some critical elliptic and parabolic problems, J. Diff. Eqs., 144 (1998), 441-476.

[11] N.GARofalo AND D.M. NhiEu, Isoperimetric and Sobolev inequalities for Carnot-Carathèodory spaces and the existence of minima surfaces, Comm. Pure Appl. Math., 49 (1996), 1081-1144.

[12] N. GAROFALO, Unique continuation for a class of elliptic operators which degenerate on a manifold of arbitrary codimension, J. Diff. Eqs., 104 (1993), 117-146.

[13] A. LoIUdICE, Sobolev inequalities with remainder terms for sublaplacians and other subelliptic operators, NoDEA Nonlinear Differ. Eqs. Appl., 13 (2006), 119-136.

[14] P. NiU, J. Dou AND H. ZHANG, Nonexistence of weak solutions for the p-degenerate subelliptic inequalities constructed by the Generalized Baouendi-Grushin vector fields, Georgian Math. J., 12, 4 (2005), 727-742.

[15] H. Zhang AND P. Niu, Picone identity and Hardy inequality for a class of vector fields, J. Math., 23 (2003), 121-125 (in Chinese). 